Research Article

\title{
Stiffness Analysis of Rectangular Isolators Reinforced by Engineering Plastics
}

\author{
Han Liu $\mathbb{D}^{1}$ Ping Tan $\mathbb{D}^{2},{ }^{2}$ and Fulin Zhou $\mathbb{D}^{1,2}$ \\ ${ }^{1}$ School of Civil Engineering, Wuhan University, Wuhan 430072, China \\ ${ }^{2}$ Cultivation Base for State Key Laboratory for Seismic Control and Structural Safety, Guangzhou University, \\ Guangzhou 510000, China
}

Correspondence should be addressed to Fulin Zhou; zhoufl@cae.cn

Received 18 December 2018; Accepted 7 March 2019; Published 28 April 2019

Academic Editor: Nuno M. Maia

Copyright (c) 2019 Han Liu et al. This is an open access article distributed under the Creative Commons Attribution License, which permits unrestricted use, distribution, and reproduction in any medium, provided the original work is properly cited.

\begin{abstract}
A novel cost-effective isolator reinforced by engineering plastics has been designed and manufactured for seismic protection for low-rise buildings in less developed areas. The reinforcement is flexible in tension, which is similar to fiber-reinforced isolators. However, available solutions for fiber-reinforced isolators are not applicable, because the Poisson effect of engineering plastics cannot be neglected, which is done for fiber reinforcement. In this paper, analytical solutions for compression and bending stiffness for rectangular isolators reinforced by engineering plastics are proposed, with both the Poisson effect of the reinforcement and the effect of rubber compressibility taken into consideration. Then, the simplified solutions are also derived, which can greatly improve calculation efficiency. To validate the solutions, finite element analysis is conducted on a set of isolators with different reinforcement stiffnesses. The results show the superiority of the proposed solutions to the previous solutions for fiber-reinforced isolators. A series of experimental tests of the isolators are also carried out to verify the solutions. Both the analytical and the simplified solutions match well with the experimental results.
\end{abstract}

\section{Introduction}

Recent earthquakes in China such as Wenchuan and Yushu earthquakes caused numerous loss of lives, which has emphasized that a greater lose may happen when earthquakes occur in rural areas and less developed areas. Ge et al. [1] investigated the buildings in these areas to find that most of them are properly designed masonry structures with insufficient seismic capacity. About $80 \%$ land of China is located in seismically active zones with an intensity magnitude of six or above, and $58 \%$ building area locates in rural areas, according to the statistics by Cao et al. [2]. These buildings need to be protected to mitigate potential risk due to severe earthquakes.

Zhou [3] systematically introduced the method for seismic protection, and one of the most effective techniques is seismic isolation technology. However, conventional isolators are expensive, large, and heavy. Kelly [4] developed fiber-reinforced isolators employing fibers to replace steel plates in laminated rubber isolators, which could reduce both the weight and the cost. In these isolators, the connecting plates are removed, which leads to an unbonded boundary condition. Kelly [5] and Kelly and Takhirov [6] made experimental attempts to prove that such kind of isolators could be produced and could provide enough capacity and proper stiffness. Unfortunately, the fibers have no flexure rigidity to restrain the rollover deformation of the isolator. Toopchi-Nezhad et al. [7] observed the rollover deformation of rectangular fiber-reinforced isolators. When it happened, the horizontal stiffness decreased apparently. Kelly and Calabrese [8] derived that the displacement of this kind of isolators was limited to 1.67 times the height of the isolator. Angeli et al. [9] tested 17 pairs of isolators and proposed the deformed pattern of the roll-off deformation. The horizontal deformation is limited as long as the free surfaces contact with the loading plane. Pauletta et al. [10] observed an unstable roll-out deformation of which the force- 
displacement curves had negative slopes. As a promotion, Tan et al. [11] proposed an innovative isolator which employed engineering plastics as reinforcement. Xu et al. [12] conducted systematical experiments of the isolators, employing two different kinds of plastic material. It was proved that engineering plastics have much higher out-ofplane stiffness than fibers, leading to higher stiffness and better ultimate properties of the isolator. As the mechanic assumptions of engineering plastics are different from fiber layers, available stiffness formulas of fiber-reinforced isolators are not appropriate to calculate this kind of isolators.

One of the earliest theoretical analyses of bonded rubber was conducted by Rocard [13] using energy method. Solutions of strip isolators and circular isolators were derived by Gent and Lindley [14] with the incompressibility assumption of the rubber. This solution was extended by Gent and Meinecke [15] to compression and bending stiffness of rectangle isolators. Koh and Lim [16] considered rubber compressibility for rectangular isolators and found that the incompressible assumption overestimated the stiffness of the isolators considerably. Tsai and Pai [17] proposed simplified solutions for circular-, square-, and strip-shaped isolators based on analytical solutions which considered rubber compressibility. All the above solutions were based on the assumption that the reinforcements were rigid.

Fiber reinforcement is flexible in tension, so the problem becomes much more complex. A widely acceptable method to simplify the problem is the pressure solution method introduced by Kelly [18]. The normal stresses are assumed to be equal, and some of the shear stress components are assumed to be zero. Based on the method, several analytical solutions for flexible reinforcement isolators have been developed. Kelly and Takhirov [6] derived stiffness of strip-type isolators considering reinforcement extensibility, but the solution was exact only for plane problems. Tsai and Kelly [19] studied the compression and bending modulus of rectangular isolators based on the assumption of rubber incompressibility. Kelly and Calabrese [20] solved the stiffness solutions, considering both reinforcement extension and rubber compressibility for circular isolators. Angeli et al. [9] further derived the solutions for rectangular isolators, but the Poisson effect of the reinforcement is neglected. They considered the fibers laid out in two orthogonal directions and simply overlapped, and the fibers did not sustain shear stress. Pinarbasi and Mengi [21] solved the stress and modulus solutions of fiber-reinforced isolators without the assumptions of pressure solution method. But the equations were complex, and only the solutions for strip-shaped isolators were achieved.

In engineering practice, even though the value of Poisson's ratio of rubber may be very close to 0.5 , the effect of compressibility is considerable to isolator stiffness and should not be neglected. For engineering plastics, the shear stress and Poisson effect cannot be neglected either. In this paper, the solutions for compression and bending stiffness of rectangular isolators reinforced by engineering plastics considering both rubber compressibility and reinforcement Poisson effect is presented. The derived solutions are expressed in terms of Fourier series which are complex and to the disadvantage of popularizing isolation technology. Thus, the weighted residual approach is introduced to solve the partial differential equation of the stress, and a semianalytical solution in a much simpler form is achieved. To validate the solutions, experimental studies are conducted, and finite element analysis is also carried out. The proposed solutions are proved to have enough accuracy.

\section{Fiber-Reinforced Plastic Plate Isolator}

Fiber-reinforced plastic plate is usually employed as the reinforcement in the novel rectangular isolator, which is a common kind of engineering plastic and belongs to composite material. It was made up of unsaturated polyester matrix and glass fiber cloth in previous research [11]. The isolator was fabricated into a prism, as shown in Figure 1. Its dimension is decided by the demand of its mechanical properties as well as its installation convenience. The cross section of the isolator is illustrated in Figure 2.

The isolator is much cheaper and lighter than conventional steel-reinforced isolators of the same size, and the processing of plastic is much easier than steel. The isolator promotes the displacement ability of fiber-reinforced isolators. Unlike fiber-reinforced isolators, the reinforcement of this isolator is composite material made up of plastic matrix and embedded glass fiber. So, the out-of-plane stiffness of the reinforcement is much higher than fiber sheets. As a result, the rollover phenomenon is limited and the vertical surface of the isolator does not contact with the loading plane even at very large horizontal deformation. It is to be noted that the stresses in two orthogonal directions in this isolator cannot be decoupled as they are in fiberreinforced isolators because of the plastic matrix. So, the analytical deviations should consider the Poisson effect of the reinforcement.

\section{Compression Analysis of the Isolator}

3.1. Equilibrium in Rubber Layer. A fiber-reinforced plastic plate isolator is constituted of multiple layers. Assuming the stress components of each layer along the height of the isolator is uniform, a layer of rubber and two layers of plastic plates are extracted as an analytical unit. Considering the analytical unit subjected to a vertical compression load $P$, the coordinate system and its deformation under $P$ are plotted in Figure 3. The thickness of the rubber layer is $t$, and the thickness of the plastic plate layer is $t_{\mathrm{f}}$. The side length is $2 b$ along the $x$-axis and $2 a$ along the $y$-axis. The total compression of the rubber is $\Delta$. The aspect ratio is defined by $r=a / b$.

The displacements of the rubber are assumed to obey the kinematic assumption as followed by Kelly and Calabrese [20]: 


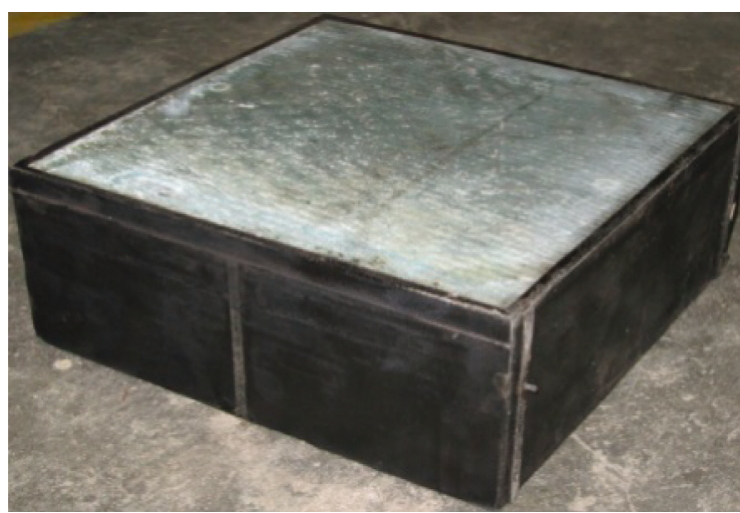

FIGURE 1: Fiber-reinforced plastic plate isolator.

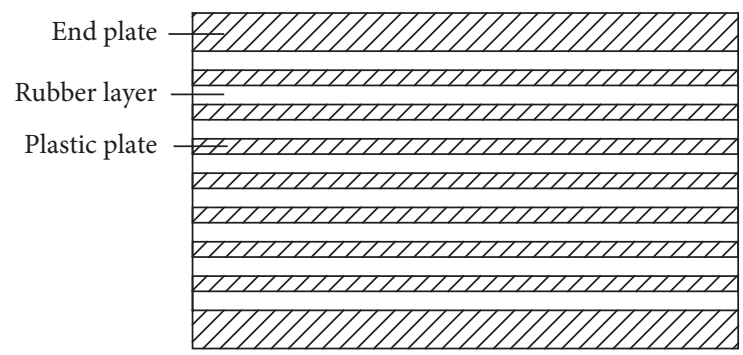

Figure 2: Cross section of the isolator.

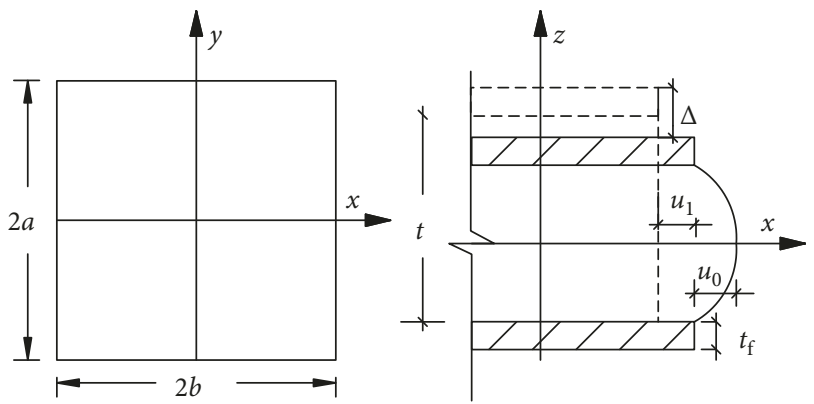

FIgURE 3: Coordinate system and deformation under compression.

$$
\begin{aligned}
& u(x, y, z)=u_{0}(x, y)\left(1-\frac{4 z^{2}}{t^{2}}\right)+u_{1}(x, y), \\
& v(x, y, z)=v_{0}(x, y)\left(1-\frac{4 z^{2}}{t^{2}}\right)+v_{1}(x, y), \\
& w(x, y, z)=w(z),
\end{aligned}
$$

where $u_{0}$ and $v_{0}$ are due to the lateral bulging of the rubber in the $x$ and $y$ directions, respectively, and $u_{1}$ and $v_{1}$ are due to the extension of the plastic plate.

The pressure solution method [18] is used here as follows:

$$
\sigma_{x x} \approx \sigma_{y y} \approx \sigma_{z z} \approx-p,
$$

where $p$ is the internal pressure. The shear stress components $\tau_{x z}$ and $\tau_{y z}$ are of order $(t / l) p$, and $\tau_{x y}$ is of order $\left(t^{2} / l^{2}\right) p^{2}$, where $l$ is a characteristic length in the $x-y$ plane and equals $2 b$ in this problem. Thus,

$$
\tau_{x y}=\tau_{y x} \approx 0 .
$$

According to the above equations and the constitutive equations of linear elastic material, there are relationships in rubber derived as follows [8]:

$$
\begin{aligned}
& \tau_{x z}=-\frac{8 G}{t^{2}} u_{0} z, \\
& \tau_{y z}=-\frac{8 G}{t^{2}} v_{0} z, \\
& p_{, x}=-\frac{8 G}{t^{2}} u_{0}, \\
& p_{, y}=-\frac{8 G}{t^{2}} v_{0} .
\end{aligned}
$$

The compressibility of rubber layer is considered by

$$
u_{, x}+v_{, y}+w_{, z}=-\frac{p}{K},
$$

where $K$ is the bulk modulus of the rubber. Substituting equations (1)-(3) into equation (10) and integrating through the thickness of the layer lead to

$$
\frac{2}{3}\left(u_{0, x}+v_{0, y}\right)+u_{1, x}+v_{1, y}=\varepsilon_{\mathrm{c}}-\frac{p}{K},
$$

where $\varepsilon_{\mathrm{c}}=\Delta / t$.

3.2. Equilibrium in Plastic Plate Layer. In a single layer of plastic plate, several layers of glass fiber cloth are embedded, of which the directions are centrosymmetric. The base material of unsaturated polyester is isotropic. Thus, the plastic plate is assumed to be an isotropic material with a modulus of $E_{\mathrm{f}}$. An analytical unit of the size $d x \times d y \times t_{\mathrm{f}}$ is extracted, and its stress state is shown in Figure 4, where $F_{x}$ and $F_{y}$ represent the normal forces of thickness $t_{\mathrm{f}}$ per unit length in $x$ and $y$ directions, and $F_{x y}$ represents the in-plane shear force. These internal forces are balanced by the shear stresses of rubber, $\tau_{x z}$ and $\tau_{y z}$, which are transmitted from adjacent layers.

The equilibrium equations in $x$ and $y$ directions, respectively, are

$$
\begin{gathered}
\frac{\partial F_{x}}{\partial x}+\frac{\partial F_{x y}}{\partial y}+\left(\left.\tau_{x z}\right|_{z=-t / 2}-\left.\tau_{x z}\right|_{z=t / 2}\right)=0 \\
\frac{\partial F_{y}}{\partial y}+\frac{\partial F_{x y}}{\partial x}+\left(\left.\tau_{y z}\right|_{z=-t / 2}-\left.\tau_{y z}\right|_{z=t / 2}\right)=0
\end{gathered}
$$

The constitutive equations considering Poisson ratio of the plates are as follows:

$$
\begin{aligned}
& F_{x}=\frac{E_{\mathrm{f}} t_{\mathrm{f}}}{1-v^{2}}\left(u_{1, x}+v v_{1, y}\right), \\
& F_{y}=\frac{E_{\mathrm{f}} t_{\mathrm{f}}}{1-v^{2}}\left(v_{1, y}+v u_{1, x}\right),
\end{aligned}
$$




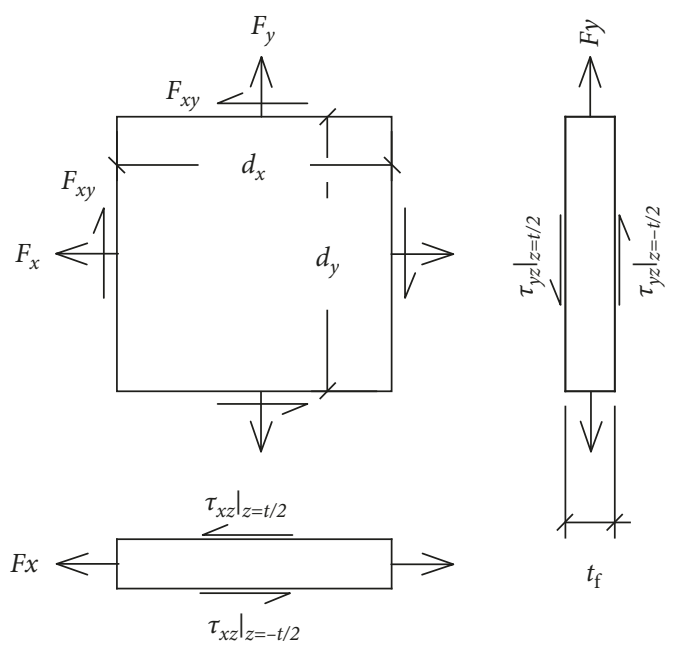

FIgURE 4: Stress components on the reinforcement.

$$
F_{x y}=\frac{E_{\mathrm{f}} t_{\mathrm{f}}}{2(1+\nu)}\left(u_{1, y}+v_{1, x}\right)
$$

where $v$ is the Poisson ratio of the plate. When $v$ equals zero, equations (14)-(16) become the same as the ones derived by Angeli et al. [9] for fiber-reinforced elastomeric bearings.

3.3. Equilibrium Equation of Pressure. The in-plane shear strain component of the rubber is as follows:

$$
\gamma_{x y}=\frac{\partial u}{\partial y}+\frac{\partial v}{\partial x} \text {. }
$$

Substituting equations (1) and (2) into equation (17), and then integrating it through the thickness, and then multiplying the result by the shear modulus $G$, the following equation is obtained:

$$
\tau_{x y}=\frac{2}{3} G\left(u_{0, y}+v_{0, x}\right)+G\left(u_{1, y}+v_{1, x}\right) .
$$

Equation (18) means $G\left(u_{1, y}+v_{1, x}\right)$ is of the same order as $\tau_{x y}$ or higher. From the pressure method assumption, as shown in equation (5), $G\left(u_{1, y}+v_{1, x}\right)$ can be assumed to be zero. With this assumption, equation (16) is simplified and we have the following equation:

$$
\begin{gathered}
F_{x y}=0, \\
F_{x y, x}=0, \\
F_{x y, y}=0 .
\end{gathered}
$$

Substituting equations (6)-(9) and (19) into equations (12) and (13), the equilibrium equations of the plate are expressed as follows:

$$
\begin{aligned}
& F_{x, x}=t p_{, x}, \\
& F_{y, y}=t p_{, y} .
\end{aligned}
$$

It is to be noted that the rubber and plastic plate layers are free in the $x-y$ plane. Thus, there are boundary conditions of $\quad p( \pm a, y)=0 ; \quad p(x, \pm b)=0 ; \quad F_{x}( \pm a, y)=0 ; \quad$ and $F_{y}(x, \pm b)=0$.

Integrating equations (20) and (21) with respect to $x$ and $y$, respectively, and then substituting boundary conditions into them, the following equations are obtained:

$$
\begin{aligned}
& F_{x}=t p, \\
& F_{y}=t p .
\end{aligned}
$$

Combining equations (14), (15), (22), and (23) leads to

$$
u_{1, x}+v_{1, y}=\frac{2(1-v)}{E_{\mathrm{f}} t_{\mathrm{f}}} t p \text {. }
$$

Differentiating equations (8) and (9) with respect to $x$ and $y$, respectively, and adding them up, and substituting the sum and equation (24) into equation (11) leads to

$$
\nabla^{2} p-\left(2 \alpha_{1}^{2}+\beta^{2}\right) p+\beta^{2} \varepsilon_{\mathrm{c}}=0,
$$

where $\nabla^{2}$ is the Laplace operator (i.e., $\nabla^{2} p=\left(\partial^{2} p / \partial x^{2}\right)+$ $\left.\left(\partial^{2} p / \partial y^{2}\right)\right)$ and

$$
\begin{aligned}
\alpha_{1} & =\sqrt{\frac{12(1-\nu) G}{E_{\mathrm{f}} t_{\mathrm{f}} t}}, \\
\beta & =\sqrt{\frac{12 G}{K t^{2}}} .
\end{aligned}
$$

The Poisson ratio of the reinforcement and the bulk modulus of rubber are both included in the equilibrium equation (25), which is derived for the first time for fiberreinforced plastic plate rectangular isolators.

3.4. Solution of Compression Modulus. The method of separation of variables is used here combining with Fourier transformation to solve equation (25). It is observed that equation (25) is in the same form as the equilibrium function derived by Angeli et al. [9]. The difference is the expression of parameter $\alpha_{1}$. Thus, the analytical solution of pressure can be solved and expressed in the same form:

$$
\begin{aligned}
p(x, y)= & \frac{192 G \varepsilon_{c}}{\pi^{2} t^{2}} \sum_{n, m=1}^{\infty} \frac{1}{m n} \sin \left(\frac{m \pi}{2}\right) \\
& \cdot \frac{\sin (n \pi / 2) \cos ((n \pi / 2 b) x) \cos ((m \pi / 2 a) y)}{(n \pi / 2 b)^{2}+(m \pi / 2 a)^{2}+\left(2 \alpha_{1}^{2}+\beta^{2}\right)} .
\end{aligned}
$$

Considering the vertical normal stress of the plate, there is a relationship as follows:

$$
\iint_{\Omega} p(x, y) d x d y=P
$$

where $P$ is the vertical resultant load. Combined with $E_{\mathrm{c}}=P /\left(A \varepsilon_{\mathrm{c}}\right)$, the compression modulus is solved and normalized as follows: 


$$
\begin{aligned}
\frac{E_{\mathrm{c}}}{G S^{2}}= & \frac{768(1+(1 / r))^{2}}{\pi^{4}} \\
& \cdot \sum_{n, m=1}^{\infty} \frac{1}{m^{2} n^{2}} \frac{\sin ^{2}(m \pi / 2) \sin ^{2}(n \pi / 2)}{\left[(n \pi / 2)^{2}+(m \pi / 2 r)^{2}+2\left(\alpha_{1} b\right)^{2}+(\beta b)^{2}\right]}
\end{aligned}
$$

The normalized compression modulus $E_{\mathrm{c}} / G S^{2}$ versus $\alpha_{1} b$ and $\beta b$ for a square case $r=1$ is plotted in Figure 5(a). For given $G$ and $S$, the compression modulus decreases obviously as either $\alpha_{1} b$ or $\beta b$ increases. For clarification, a parameter $\alpha$ is defined as $\alpha=\sqrt{2 /(1-\nu)} \alpha_{1}$. The variation of normalized compression modulus $E_{\mathrm{c}} / G S^{2}$ with Poisson ratio $\nu$ for different values of $\beta b$ is plotted in Figure 5(b). The value of $E_{\mathrm{c}} / G S^{2}$ at $\nu=0$ means the modulus ignoring the effect of the Poisson ratio, which corresponds to the solutions by Angeli et al. [9]. It can be observed that, for smaller value of $\beta b, E_{\mathrm{c}} / G S^{2}$ is more sensitive to the change of $\nu$. Figure $5(\mathrm{c})$ shows $E_{\mathrm{c}} / G S^{2}$ as a function of $\beta b$ with $\nu=0.3$ for different values of $\alpha b$. For both rigid reinforcement with $\alpha b=0$ and very flexible reinforcement with $\alpha b=3, E_{\mathrm{c}} / G S^{2}$ is very sensitive to $\beta b$. As $\beta b$ is a function of $K$, it is obvious that the compressibility of rubber is not negligible. The curves of $E_{\mathrm{c}} / G S^{2}$ versus $r$ for different values of $\beta b$ are plotted in Figure 5(d), which reveals that the normalized compression modulus of square type is the largest among rectangle isolators.

3.5. Simplified Solution of Compression Modulus. To simplify the solution of the square isolator, a numerical method of weighted residuals (MWR) introduced by $\mathrm{Xu}$ [22] is used to solve equation (25). After several tests, a polynomial $p_{1}(x, y)$ is selected as a trial function of pressure $p$ for $r$ near 1 and automatically satisfies boundary conditions as follows:

$$
p_{1}(x, y)=C_{1}\left(x^{2}+C_{2}\right) \cdot\left(x^{2}-b^{2}\right)\left(y^{2}+C_{3}\right)\left(y^{2}-a^{2}\right) .
$$

Replacing $p(x, y)$ with $p_{1}(x, y)$ in equation (25), the left-hand side of the equation does not equal to zero in the whole domain, and the residual is defined by $R(x, y)$ as follows:

$$
R(x, y)=\nabla^{2} p_{1}-\left(2 \alpha_{1}^{2}+\beta^{2}\right) p_{1}+\frac{12 G}{t^{2}} \varepsilon_{c} .
$$

Using collocation method to eliminate the residuals by

$$
R\left(x_{i}, y_{i}\right)=0, \quad i=1,2, \ldots, N,
$$

where $\left(x_{i}, y_{i}\right)$ represents a point in the definition domain, and $N$ is the number of such points, which can be determined by the number of undetermined coefficients in above equations. The selection of $\left(x_{i}, y_{i}\right)$ is very important for the solution accuracy. After tests, five points at $(0,0),( \pm b$, $0)$, and $(0, \pm a)$ are selected which can lead to three equations due to symmetry. In order to solve simplified modulus $E_{\mathrm{cs}}$, an additional equation is given by assuming that $p_{1}(x, y)$ satisfies equation (28). Then, $C_{i}$ and $E_{\mathrm{cs}}$ can be solved as follows:

$$
\begin{aligned}
C_{1}= & \frac{225 P}{16 a^{10}} \\
& \cdot \frac{r^{5}\left(5 \gamma^{2}+12-\left(2 / r^{2}\right)\right)\left(5 \gamma^{2}-2+\left(12 / r^{2}\right)\right)}{\left(5 \gamma^{2}+12+\left(348 / r^{2}\right)\right)\left(5 \gamma^{2}+348+\left(12 / r^{2}\right)\right)}, \\
C_{2}= & \frac{70 a^{2}}{r^{2}\left(5 \gamma^{2}-2+\left(12 / r^{2}\right)\right)}, \\
C_{3}= & \frac{70 a^{2}}{r^{2}\left(5 \gamma^{2}+12-\left(2 / r^{2}\right)\right)}, \\
\frac{E_{c s}=}{G S^{2}}= & \frac{4(1+r)^{2}}{13125} \\
& \cdot \frac{\left(5 \gamma^{2}+12+\left(348 / r^{2}\right)\right)\left(5 \gamma^{2}+348+\left(12 / r^{2}\right)\right)}{\left(5 \gamma^{2}+12+\left(12 / r^{2}\right)\right)},
\end{aligned}
$$

where $\gamma$ is a dimensionless parameter defined by the following equation:

$$
\gamma^{2}=\left(2 \alpha_{1}^{2}+\beta^{2}\right) b^{2}
$$

For a special case of a square isolator with $r$ equals 1 , equation (36) reduces to

$$
\frac{E_{\mathrm{cs}}}{G S^{2}}=\frac{16}{525} \frac{\left(\gamma^{2}+72\right)^{2}}{5 \gamma^{2}+24} \text {. }
$$

$E_{\mathrm{cs}} / G S^{2}$ is plotted versus $\alpha_{1} b$ and $\beta b$ in Figure 6(a). It has similar shape as the analytical solution plotted in Figure 5(a). It is to be noted that the simplified solution has enough accuracy only when the polynomial assumption of pressure $p$ in equation (30) approaches its real distribution. $E_{\mathrm{cs}} / E_{\mathrm{c}}$ is plotted versus $\gamma$ in Figure 6(b). The error of simplified solution increases as $\gamma$ increases. It is observed that when $\gamma$ is smaller than 2.60, which covers most of the square fiberreinforced plastic plate isolators, the error of simplified compression modulus is smaller than $10 \%$. A more accurate simplified solution can be obtained by improving the order of the trial function of $p$ in equation (30). For isolators with other aspect ratios, a simplified solution can also be obtained by this method on the basis of a proper selection of the trial function of $p$.

3.6. Comparison with Finite Element Analysis. A special case of square cross section with typical geometry parameters is considered. Six models with different elastic moduli of the reinforcement $E_{\mathrm{f}}$ are established within the environment of ABAQUS. All models have a side length of $230 \mathrm{~mm}$ and a total thickness of $11 \mathrm{~mm}$. The thickness of the reinforcement layer and the rubber layer is $t_{\mathrm{f}}=3 \mathrm{~mm}$ and $t=5 \mathrm{~mm}$, respectively. A constant value of Poisson's ratio of 0.3 is used for the reinforcement material. The rubber had a shear modulus of $G=0.46 \mathrm{MPa}$ and bulk modulus of $K=2040 \mathrm{MPa}$. $E_{\mathrm{f}}$ varies from $0.5 \mathrm{GPa}$ to $20 \mathrm{GPa}$, and the resulting $\gamma$ distribute in a relatively large range from 1.33 to 3.88, as shown in Table 1. 

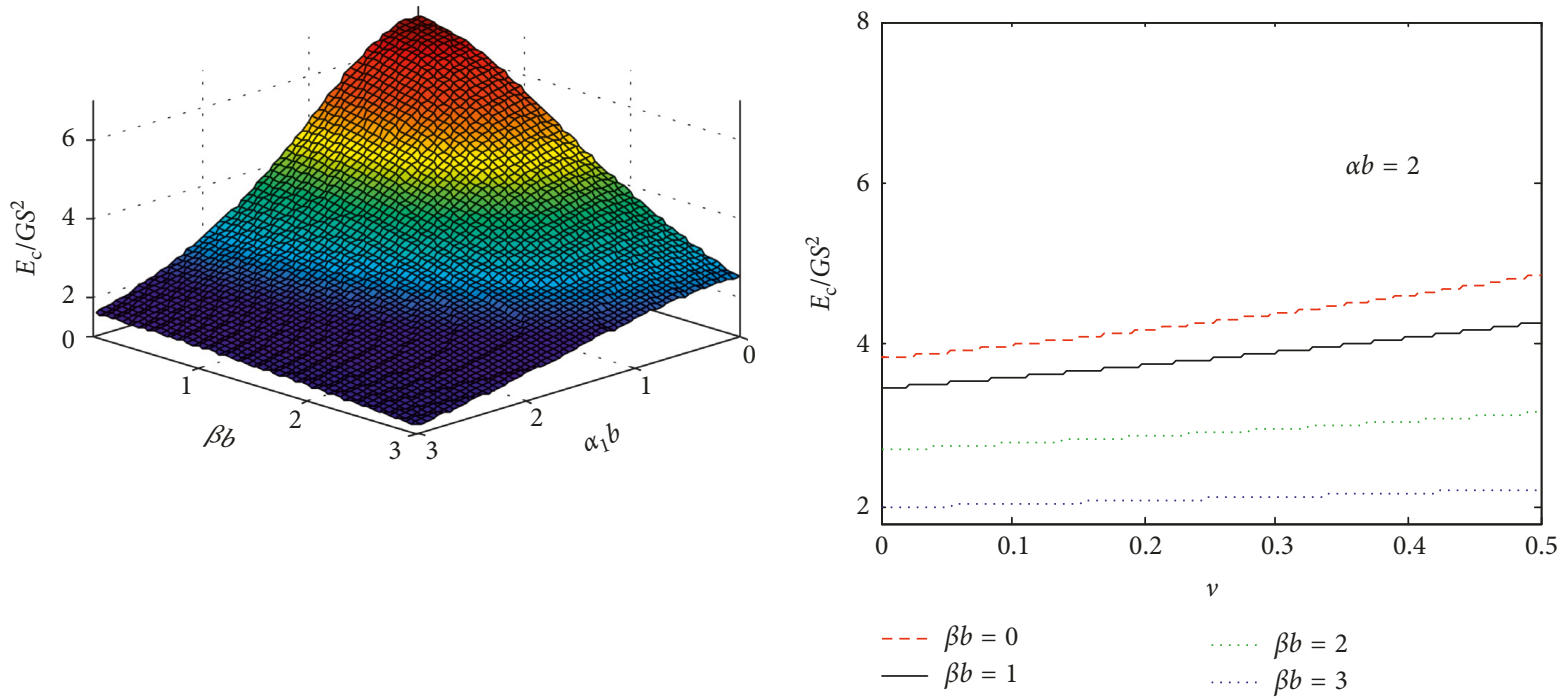

(a)

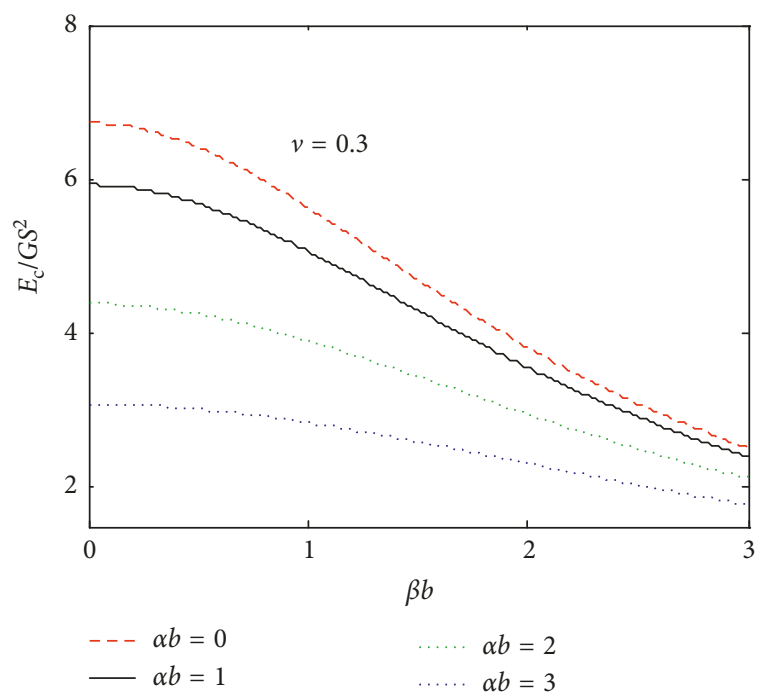

(c)

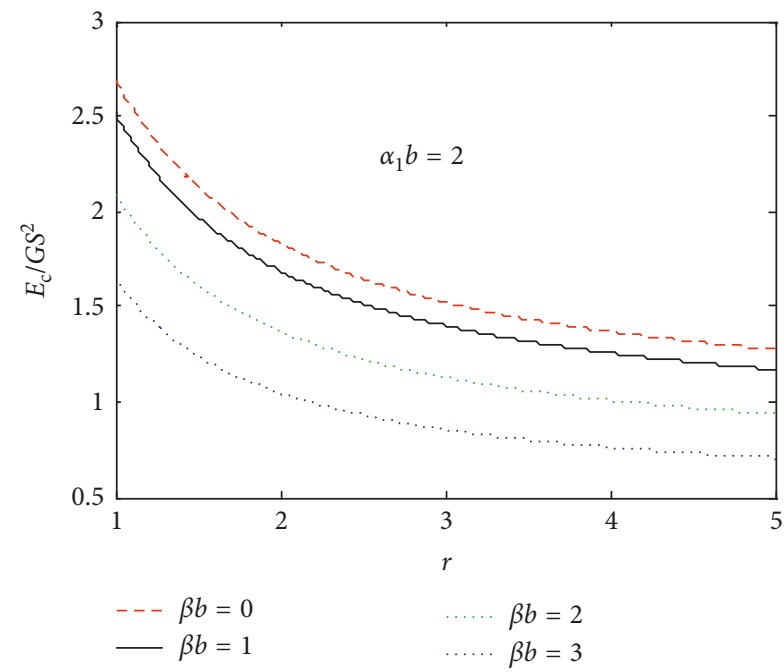

(d)

Figure 5: Parameter analysis of $E_{c^{\prime}}$ (a) $E_{c}$ of a square case. (b) Effect of Poisson ratio. (c) Effect of $\beta b$. (d) Effect of aspect ratio.

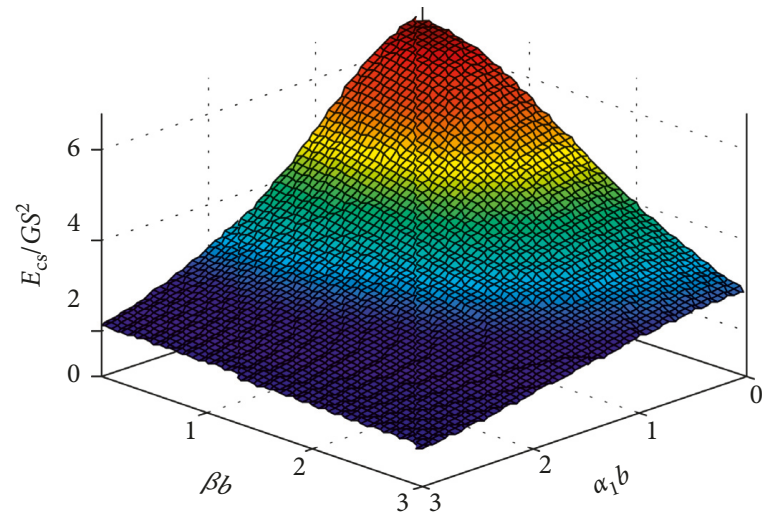

(a)

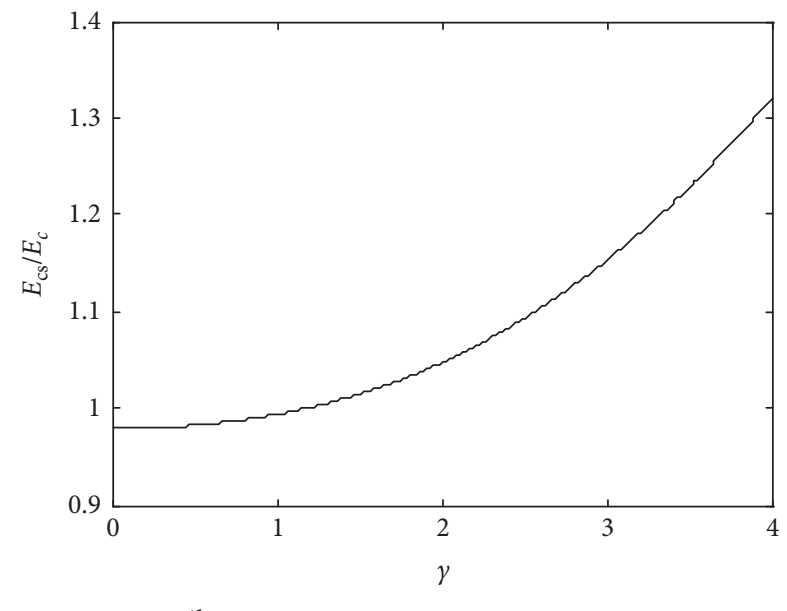

(b)

Figure 6: Simplified solution for $E_{\mathrm{c}}$ of a square case. (a) Simplified solution. (b) Error analysis. 
TABle 1: Parameters of the models.

\begin{tabular}{lccc}
\hline Model & $E_{\mathrm{f}}(\mathrm{GPa})$ & $\alpha_{1} b$ & $\gamma$ \\
\hline $\mathrm{P}_{1}$ & 0.5 & 2.61 & 3.88 \\
$\mathrm{P}_{2}$ & 1 & 1.85 & 2.87 \\
$\mathrm{P}_{3}$ & 2 & 1.31 & 2.20 \\
$\mathrm{P}_{4}$ & 5 & 0.83 & 1.67 \\
$\mathrm{P}_{5}$ & 10 & 0.58 & 1.45 \\
$\mathrm{P}_{6}$ & 20 & 0.41 & 1.33 \\
\hline
\end{tabular}

The Ogden model was used to describe the hyperelastic behavior of rubber. Reinforcement material was modeled by linear elastic isotropic material. The out-of-plane translational degrees of freedom and the rotational degrees of freedom of the reinforcement layers were restrained by reference points to keep the layer plain. The in-plane translational degrees of freedom were released to allow the in-plain deformation. The meshing is shown in Figure 7. A vertical pressure of $5 \mathrm{MPa}$ is applied. The results of compression modulus are compared with the solutions proposed in this paper, the solutions for incompressiblerubber isolators by Tsai and Kelly [19] and the solutions for fiber-reinforced isolators by Angeli et al. [9], as shown in Figure 8.

It is observed that the proposed analytical and simplified solutions always present better evaluation of $E_{\mathrm{c}} / G S^{2}$ than other solutions, except for model $\mathrm{P}_{6}$. When $\alpha_{1} b$ is very small, the stiffness of the reinforcement is high, and the proposed solutions tend to underestimate the constraint effect of the reinforcement. However, $E_{\mathrm{f}}$ of $\mathrm{P}_{6}$ is as high as steel, which is almost impossible for a plastic plate employed in this isolator. In other points, the analytical curve matches well with FEA results. The error of the simplified solution becomes obvious when $\alpha_{1} b$ is large, but it is still smaller than previous solutions and can be optimized as discussed in Section 3.5. The proposed solutions coincide with Angeli solution at $\alpha_{1} b=0$, because the reinforcement is strictly rigid and the effect of Poisson ratio vanishes. When $\alpha_{1} b$ is larger than 0 , Angeli solution always underestimates the modulus, showing that the effect of the Poisson ratio cannot be neglected. It can also be seen that Tsai solution always overestimates the compression modulus, showing that the effect of the compressibility of rubber need to be included.

3.7. Compression Stiffness. For an isolator with $n$ layers, the effective compression modulus of the isolator is [23]

$$
\left(E_{\mathrm{c}}\right)_{\text {free }}=\frac{n}{\left(2 / E_{\mathrm{c}}^{1}\right)+\left((n-2) / E_{\mathrm{c}}\right)},
$$

where $E_{\mathrm{c}}^{1}$ is the modulus of exterior layers. When $n$ is big, $\left(E_{\mathrm{c}}\right)_{\text {free }}$ is assumed to be equal to $E_{\mathrm{c}}$ [23]. The vertical stiffness can be calculated using equation (29):

$$
K_{\mathrm{v} . \mathrm{ana}}=\frac{3072 G a b^{3}}{n \pi^{4} t^{3}} \cdot \sum_{n, m=1}^{\infty} \frac{\sin ^{2}(m \pi / 2) \sin ^{2}(n \pi / 2)}{m^{2} n^{2}\left[(n \pi / 2)^{2}+(m \pi / 2 r)^{2}+\gamma^{2}\right]} .
$$

The simplified form is calculated using equation (36):

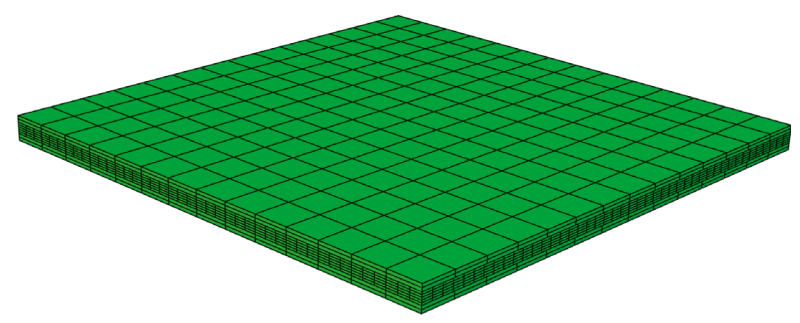

Z. Step: Step-1

Figure 7: FEA model and meshing.

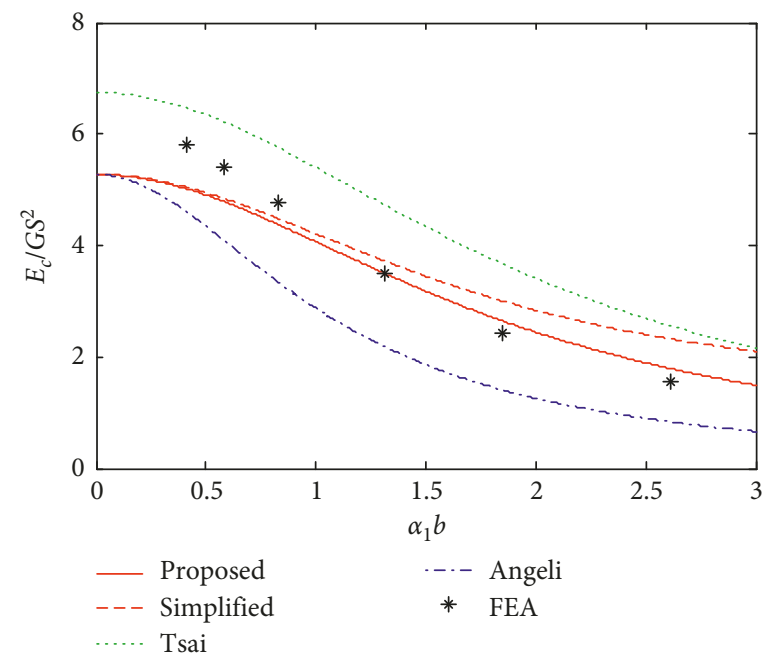

Figure 8: Comparison of $E_{\mathrm{c}} / G S^{2}$.

$$
\begin{aligned}
K_{\mathrm{v} . \operatorname{sim}}= & \frac{16 G a^{3} b}{13125 n t^{3}} \\
& \cdot \frac{\left(5 \gamma^{2}+12+\left(348 / r^{2}\right)\right)\left(5 \gamma^{2}+348+\left(12 / r^{2}\right)\right)}{\left(5 \gamma^{2}+12+\left(12 / r^{2}\right)\right)} .
\end{aligned}
$$

\section{Bending Analysis of the Isolator}

Applying moment $M$ about $y$-axis to the analytical unit, the resultant deformation is illustrated in Figure 9, in which the out-of-plane deformation of the reinforcement is neglected to simplify the analysis.

4.1. Equilibrium Equation of Pressure. The displacement functions of rubber in the $x-y$ plane are the same as the compression condition and are expressed by equations (1) and (2), respectively. In $z$ direction, it is expressed by

$$
w(x, y, z)=\theta z \frac{x}{t} .
$$

The equilibrium of stress in rubber taking into account of rubber compressibility in a pure bending condition is derived by Angeli et al. [9], and there are two equations: 


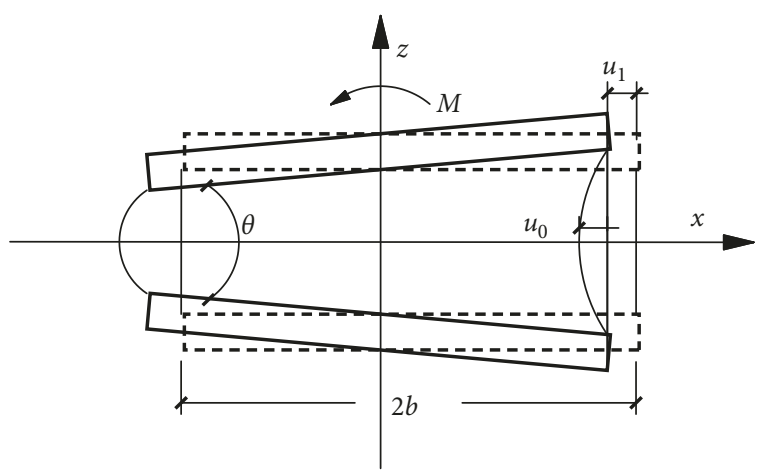

Figure 9: Deformation under bending.

$$
\begin{aligned}
\frac{2}{3}\left(u_{0, x}+v_{0, y}\right)+u_{1, x}+v_{1, y} & =-\frac{\theta x}{t}-\frac{p}{K} \\
p_{, x} & =-\frac{8 G}{t^{2}} u_{0}+\frac{G \theta}{t} .
\end{aligned}
$$

In addition, the expression of $p_{, y}$ is the same as equation (9). Although the load of the bending condition is different from the compression condition, the equilibrium and boundary conditions of stresses in the reinforcement layer remain the same. Thus, equation (24) is also tenable in the bending problem. Substituting equations (9), (24), and (44) in (11), the following equation is obtained:

$$
\nabla^{2} p-\left(2 \alpha_{1}^{2}+\beta^{2}\right) p=-\frac{12 G}{t^{2}} \frac{\theta x}{t}
$$

This equilibrium equation of $p$ under pure bending is derived for the first time for fiber-reinforced plastic plate rectangular isolators.

4.2. Solution of Bending Modulus. Equation (45) is in the same form as the equilibrium equation derived by Angeli et al. [9] except the expression of $\alpha_{1}$. A similar procedure of solving this equation is conducted employing the method of separation of variables. The following equation is obtained:

$$
\begin{aligned}
p(x, y)= & \frac{96 G b \theta}{\pi^{3} t^{3}} \sum_{n, m=1}^{\infty} \frac{1}{m n^{2}} \\
& \cdot \frac{\sin (m \pi / 2)[n \pi \cos (n \pi)-\sin (n \pi)]}{(n \pi / b)^{2}+(m \pi / 2 a)^{2}+\left(2 \alpha_{1}^{2}+\beta^{2}\right)} \\
& \cdot \sin \left(\frac{n \pi}{b} x\right) \cos \left(\frac{m \pi}{2 a} y\right) .
\end{aligned}
$$

The applied moment $M$ can be expressed as follows:

$$
M \approx-\int_{-b}^{b} \int_{-a}^{a} p(x, y) x d x d y .
$$

Combining with $E_{\mathrm{b}}=M / I(\theta / t)$, where $I$ is the moment of inertia about the $y$-axis, the bending modulus is solved and normalized as follows:

$$
\frac{E_{\mathrm{b}}}{G S^{2}}=\frac{576(1+(1 / r))^{2}}{\pi^{4}} \cdot \sum_{n, m=1}^{\infty} \frac{\sin ^{2}(m \pi / 2)}{m^{2} n^{2}\left[(n \pi)^{2}+(m \pi / 2 r)^{2}+\gamma^{2}\right]} .
$$

The normalized bending modulus $E_{\mathrm{b}} / G S^{2}$ versus $\alpha_{1} b$ and $\beta b$ for a square case $(r=1)$ is plotted in Figure 10(a). For given $G$ and $S$, the bending modulus decreases obviously as either $\alpha_{1} b$ or $\beta b$ increases. A parameter analysis is conducted of Poisson ratio $\nu, \beta b$, and aspect ratio $r$, as plotted in Figures $10(\mathrm{~b})-10(\mathrm{~d})$. It can be observed that $E_{\mathrm{b}} / G S^{2}$ varies nearly linearly with the variation of Poisson ratio $v$ and $E_{\mathrm{b}} / G S^{2}$ is more sensitive to $\beta b$ than to $\nu$. The normalized bending modulus of square type is the largest among rectangle isolators of different aspect ratios.

4.3. Simplified Solution of Bending Modulus. The MWR method is also used here to achieve a simplified solution of bending modulus. After analyzing the shape of the function of $E_{\mathrm{b}}$ in equation (48) and conducting several tests of trial functions of pressure $p$, a polynomial function as shown in equation (49) is selected as a balance point between accuracy and simplification:

$$
p_{2}=D_{1} x\left(x^{2}-b^{2}\right)\left(y^{2}-a^{2}\right)\left(y^{2}+D_{2}\right) .
$$

This function automatically satisfies the boundary condition that the stresses on the boundary equal zero. Substituting it into equation (45) and eliminating the residual by collocation method at $( \pm(\sqrt{3} b / 3), 0)$, $( \pm(\sqrt{3} b / 3), \pm a)$, and $(0,0), D_{i}$ can be solved and finally we have the following equation:

$$
\frac{E_{\mathrm{bs}}}{G S^{2}}=\frac{12\left(\gamma^{2}+9+\left(60 / r^{2}\right)\right)(1+r)^{2}}{25\left(5 \gamma^{2}+45+\left(12 / r^{2}\right)\right)} \text {. }
$$

For a square case, it can be further simplified as follows:

$$
\frac{E_{\mathrm{bs}}}{G S^{2}}=\frac{48}{25} \frac{\gamma^{2}+69}{5 \gamma^{2}+57} \text {. }
$$

$E_{\mathrm{bs}} / G S^{2}$ is plotted versus $\alpha_{1} b$ and $\beta b$ in Figure 11(a). It has similar shape as the analytical solution plotted in Figure 10(a). $E_{\mathrm{bs}} / E_{\mathrm{b}}$ is plotted versus $\gamma$ in Figure 11(b). The error of simplified solution increases as $\gamma$ increases. It is observed that, when $\gamma$ is smaller than 3.4, the error of simplified bending modulus is smaller than $10 \%$, which is $30 \%$ larger than the suitable range of $E_{\mathrm{cs}}$ and can cover most of the common square isolators. A more accurate simplified solution can be obtained by improving the order of the trial function of $p$ in equation (49). For isolators with other aspect ratios, a simplified solution can also be obtained by this method on the basis of a proper selection of the trial function of $p$.

4.4. Comparison with Finite Element Analysis. The models established in section 3.6 are employed, and a rotation of $0.005 \mathrm{rad}$ is applied to the reinforcement layer to analyze the bending modulus. The FEA results are normalized and compared with the proposed solutions, Tsai solution and Angeli solution, as shown in Figure 12. 


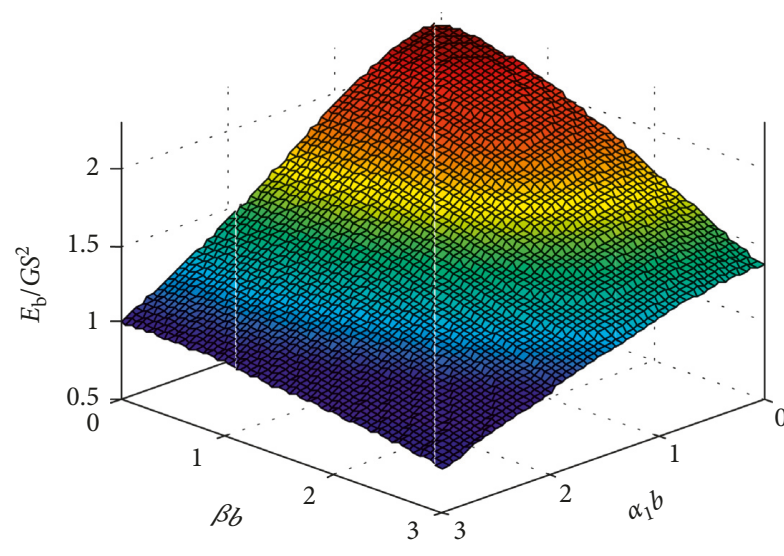

(a)

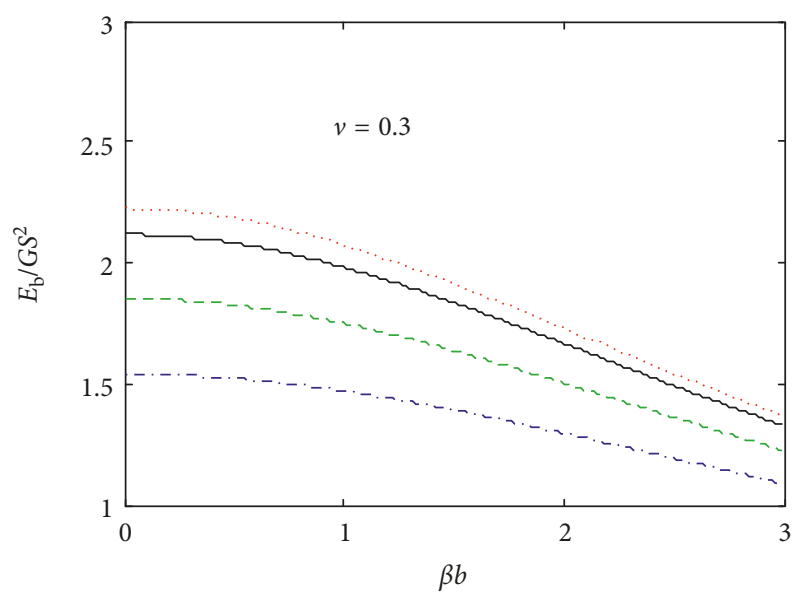

$\begin{aligned} \cdots \cdots & \alpha b=0 \\ -\alpha b & =1\end{aligned}$

$--\alpha b=2$

-.- $\alpha b=3$

(c)

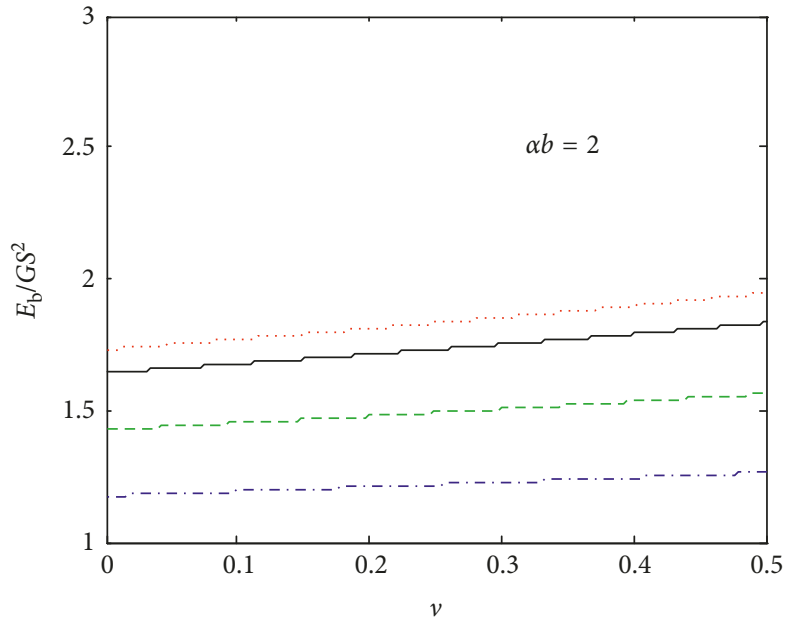

(b)

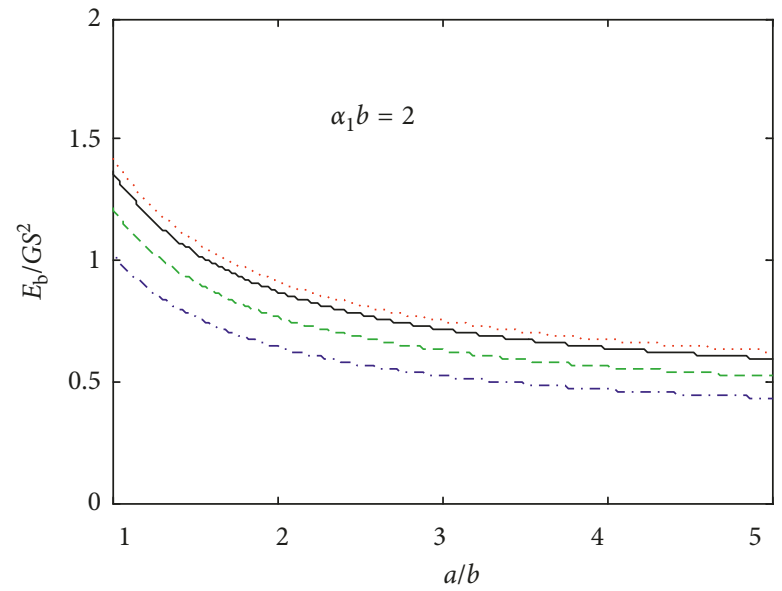
‥ $\beta b=0$
$--\beta b=2$
- $\beta b=1$
-. $\beta b=3$

(d)

Figure 10: Parameter analysis of $E_{\mathrm{b}}$. (a) $E_{\mathrm{b}}$ of a square case. (b) Effect of Poisson ratio. (c) Effect of $\beta b$. (d) Effect of aspect ratio.

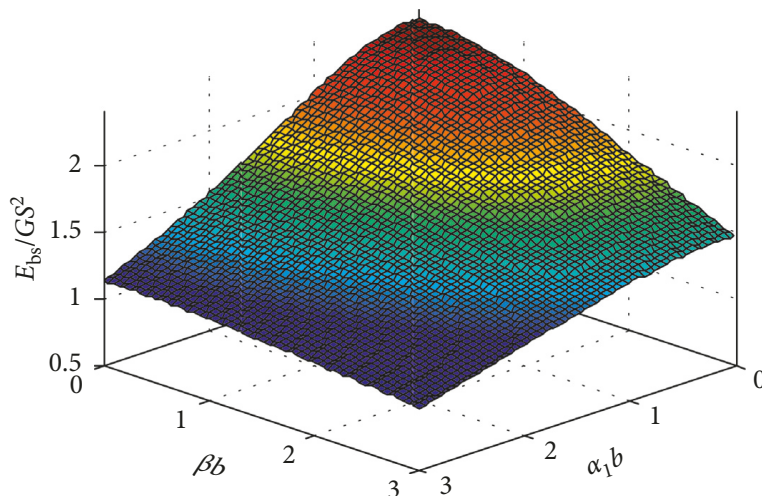

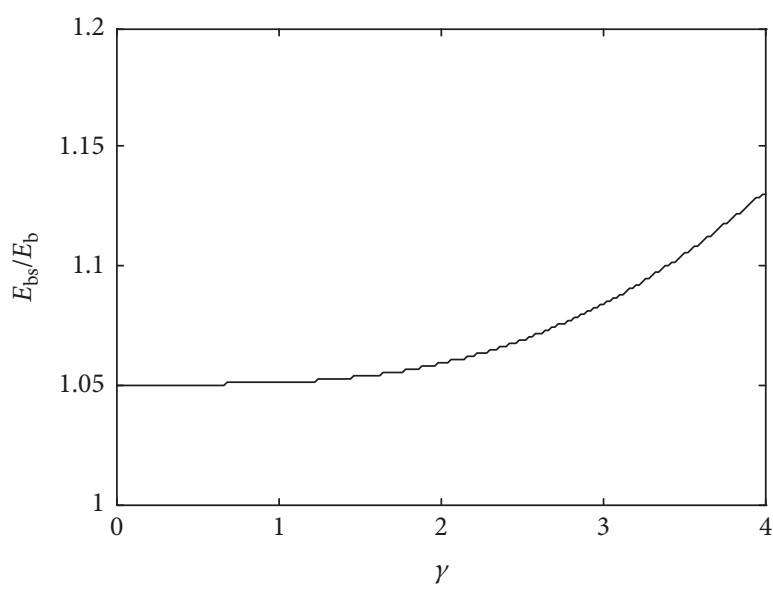

$-a / b=1$

(a)

(b)

FIgURE 11: Simplified solution for $E_{\mathrm{b}}$ of a square case. (a) Simplified solution. (b) Error analysis. 


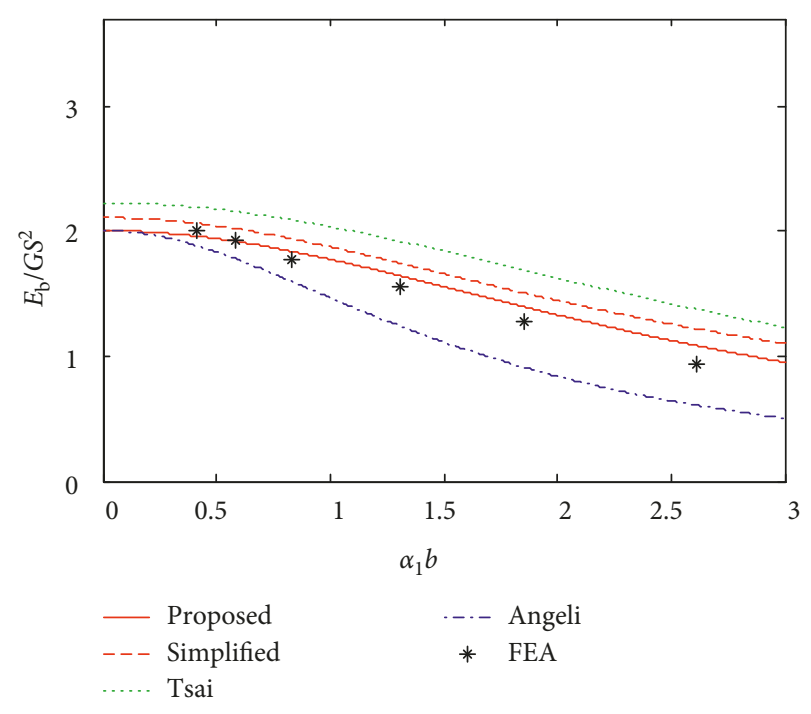

Figure 12: Comparison of $E_{\mathrm{b}} / G S^{2}$.

The validity of the proposed solutions is confirmed by the curves. Both the analytical and simplified solutions are obviously closer to the FEA results. As $E_{\mathrm{f}}$ is commonly larger than $0.5 \mathrm{GPa}$, the solutions exhibit very good approximation of the modulus at the five points from the left in Figure 12. Similar to the conclusions for the compression case, Angeli solution always underestimates the bending modulus and Tsai solution always overestimates it, showing that the effects of rubber compressibility and reinforcement Poisson ratio are also important for bending modulus.

4.5. Horizontal Stiffness. The effect of the bending modulus of a single layer on the horizontal stiffness of an isolator is considered based on the theory of Haringx [24]. A simplified formula has enough accuracy to calculate the initial effective horizontal stiffness [25]:

$$
K_{\mathrm{H}}=\frac{G A}{n t}\left(1-\left(\frac{P}{P_{\mathrm{cr}}}\right)^{2}\right)
$$

where $P_{\text {cr }}$ is the bucking load of the isolator, which has a simplified form as follows:

$$
P_{\mathrm{cr}}=\frac{\pi}{n t} \sqrt{G A E_{\mathrm{b}} I}
$$

where $E_{\mathrm{b}}$ is calculated by the proposed solutions, and $I$ is the inertia moment of the cross section.

The analytical solution of $P_{\mathrm{cr}}$ is obtained as follows:

$$
P_{\text {cr.ana }}=\frac{48 G}{n \pi t^{2}} \cdot \sqrt{\sum_{n, m=1}^{\infty} \frac{a b^{3} I \sin ^{2}(m \pi / 2)}{m^{2} n^{2}\left[(n \pi)^{2}+(m \pi / 2 r)^{2}+\gamma^{2}\right]}} .
$$

The simplified solution for $P_{\mathrm{cr}}$ is

$$
P_{\text {cr.sim }}=\frac{4 G \pi}{5 n t^{2}} \cdot \sqrt{\frac{3 a^{3} b I\left(\gamma^{2}+9+\left(60 / r^{2}\right)\right)}{\left(5 \gamma^{2}+45+\left(12 / r^{2}\right)\right)}} .
$$

The analytical and simplified solutions for horizontal stiffness can be obtained by substituting equations (54) and (55) into equation (52), respectively.

\section{Experimental Verification}

5.1. Test Specimen. Eight representative specimens were tested to verify the proposed solutions. The specimens have been designed based on commonly used materials and dimensions of fiber-reinforced plastic plate isolators. The detailed parameters are summarized in Table 2. Each specimen is described by aspect ratio $r$, elastic modulus of reinforcement $E_{\mathrm{f}}$, number of rubber layers $n$, side length $2 b$, thickness of each rubber layer $t$, thickness of each reinforcement layer $t_{\mathrm{f}}$, and shear modulus of rubber $G$. The dimensionless parameter $\gamma$ is reported in the last column.

5.2. Setup and Test Procedure. All the bearings were tested on the compression-shear testing machine in the Earthquake Engineering Research and Test Center of Guangzhou University, China. The vertical actuator can impose a maximum compression force of $10,000 \mathrm{kN}$, and the horizontal actuator is capable of a maximum force of $\pm 500 \mathrm{kN}$.

Standard cyclic vertical and horizontal tests as shown in Figure 13 were conducted according to the method prescribed in the Chinese standard [26]. The isolators were subjected to a cyclic loading for four cycles in each test, and the data of the third cycle were used for calculation. The vertical tests were conducted under a design pressure of $5 \mathrm{MPa}$ for each specimen, and the horizontal tests were loaded to a shear strain of $100 \%$ to achieve equivalent stiffness.

\subsection{Results and Discussion}

5.3.1. Vertical Tests. Samples of the results of vertical tests of $r=1$ and $r=2$ are shown in Figures 14(a) and 14(b). Specimen B3 has smaller shape factor $S$ than B5 but shows obvious larger vertical stiffness in Figure 14(a), which proves the increase of $\gamma$ results in reduction in compression modulus as derived by equations (29) and (36). The comparison between $\mathrm{B} 7$ and $\mathrm{B} 8$ shows that the increase of $G$ results in increase of compression stiffness, despite a resultant increase of $\gamma$, which indicates $G$ has a larger effect.

5.3.2. Horizontal Tests. A sample of the results of horizontal tests of B7 is shown in Figures 15(a) and 15(b). It can be observed that there is a big difference between the stiffness in $x$ and $y$ directions. This phenomenon cannot be explained by traditional stiffness formula of $G A / T_{r}$. But it is reasonable in equation (48) in which different aspect ratio $r$ leads to different bending modulus. The damping ratio of the isolator in $x$ direction is $4.0 \%$ and in $y$ direction is $3.0 \%$.

5.3.3. Discussion. The test results and analytical and simplified solutions are listed in Table 3 for $r=1$, including pressure $\sigma_{z}$, experimental vertical and horizontal stiffness 
TABLE 2: Characteristics of tested specimens.

\begin{tabular}{|c|c|c|c|c|c|c|c|c|c|}
\hline No. & Aspect ratio & $E_{\mathrm{f}}(\mathrm{MPa})$ & $n$ & $2 b(\mathrm{~mm})$ & $t(\mathrm{~mm})$ & $t_{\mathrm{f}}(\mathrm{mm})$ & $G(\mathrm{MPa})$ & $S$ & $\gamma$ \\
\hline $\mathrm{B} 1$ & & 19400 & 11 & 230 & 5 & 3 & 0.4 & 11.5 & 1.34 \\
\hline B2 & & 17400 & 11 & 230 & 5 & 3 & 0.4 & 11.5 & 1.36 \\
\hline B3 & 1 & 17400 & 12 & 230 & 4.25 & 3 & 0.4 & 13.53 & 1.56 \\
\hline B4 & & 17400 & 12 & 230 & 4.25 & 3 & 0.6 & 13.53 & 1.91 \\
\hline B5 & & 3000 & 12 & 230 & 4 & 3 & 0.4 & 14.38 & 2.10 \\
\hline B6 & & 3000 & 6 & 90 & 5 & 4 & 0.4 & 6.67 & 0.72 \\
\hline B7 & 2 & 17400 & 12 & 230 & 4.25 & 3 & 0.4 & 18.04 & 1.56 \\
\hline B8 & & 17400 & 12 & 230 & 4.25 & 3 & 0.6 & 18.04 & 1.91 \\
\hline
\end{tabular}

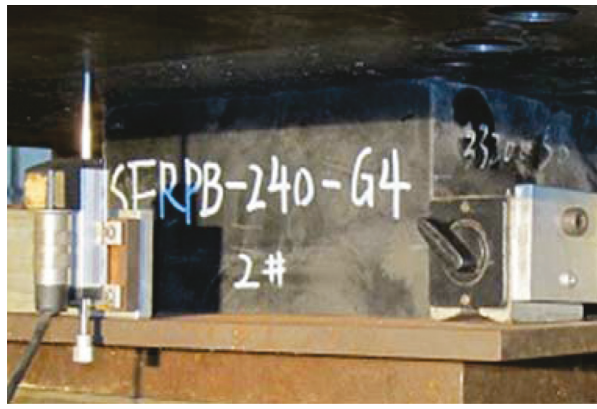

(a)

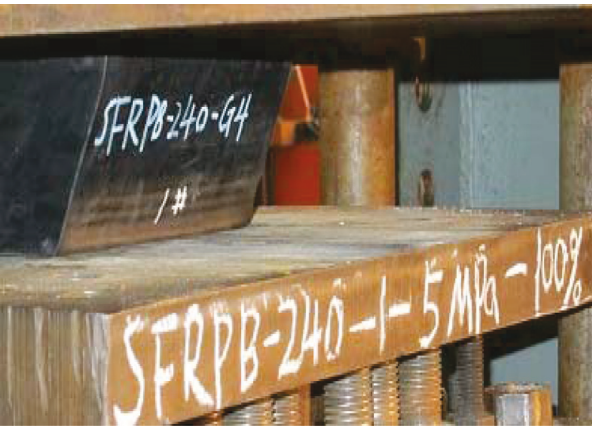

(b)

Figure 13: Experiment setup. (a) Vertical test. (b) Horizontal test.

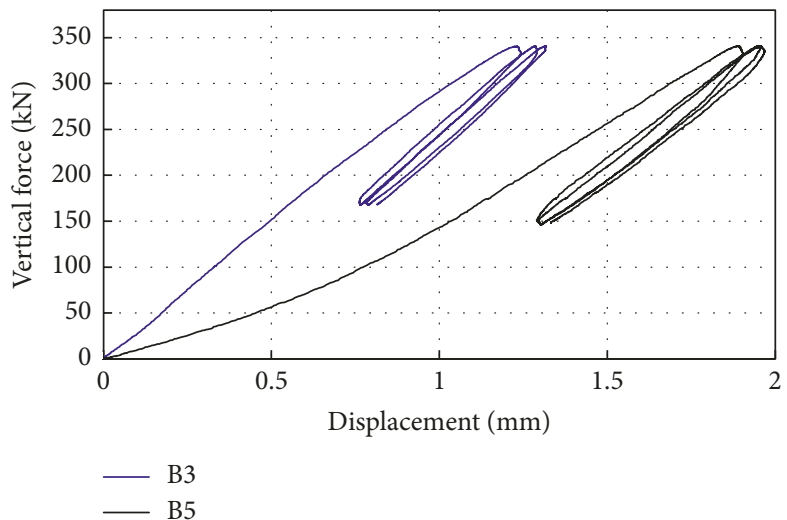

(a)

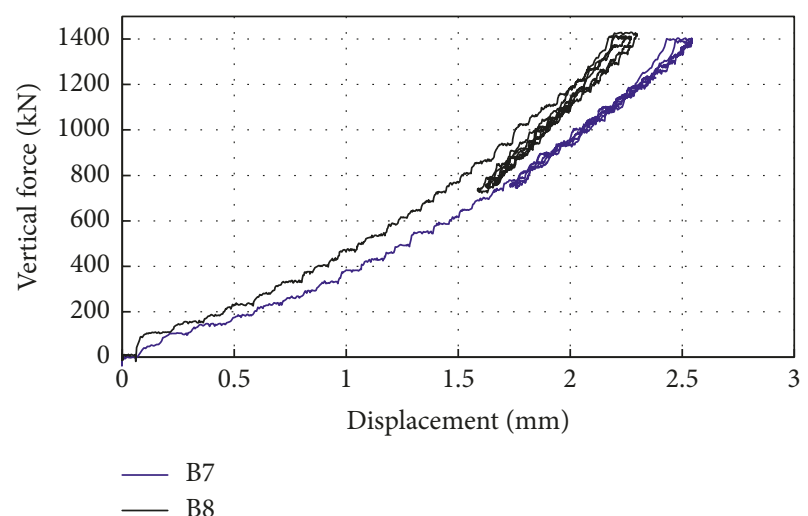

(b)

Figure 14: Vertical test results. (a) Specimens B3 and B5. (b) Specimens B7 and B8.

$K_{\text {v.exp }}$ and $K_{\text {H.exp }}$, analytical vertical and horizontal stiffness $K_{\mathrm{v} . \text { ana }}$ and $K_{\mathrm{H} . \text { ana }}$, and simplified vertical and horizontal stiffness $K_{\mathrm{v} . \text { sim }}$ and $K_{\mathrm{H} \text {.sim. }}$. Test results and analytical solutions are listed in Table 4 for $r=2$. The vertical stiffness was tested under design pressure of each specimen, and the relativity between vertical stiffness and pressure is not discussed.

As can be seen from Tables 3 and 4, results by the proposed analytical and simplified solutions agree well with the experimental results for both cases. The relative differences of analytical solutions for vertical and horizontal stiffness are less than $8.7 \%$ and $11.7 \%$, and the relative difference of simplified solutions for both vertical and horizontal stiffness is less than $11.7 \%$.

\section{Conclusion}

The analytical solutions for compression and bending modulus of rectangular fiber-reinforced plastic plate isolators are proposed, and simplified solutions are also derived for a special case of square shape. The solutions show advantages to the existing solutions and agree well with experimental tests.

Based on the analytical analysis, finite element analysis, and experimental results, the following conclusions can be made:

(1) The effects of the Poisson ratio of reinforcement and compressibility of rubber cannot be neglected for both compression and bending modulus. The 


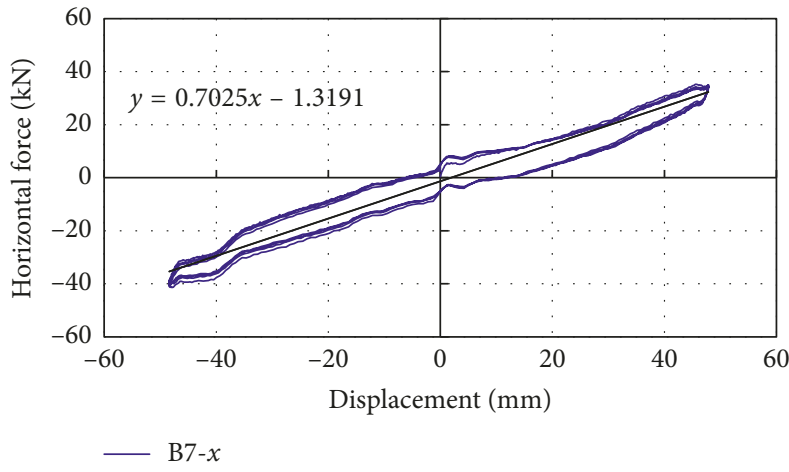

(a)

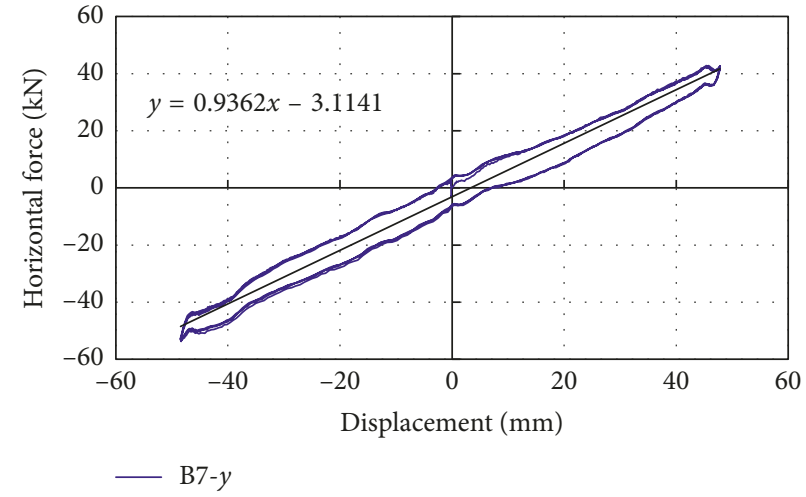

(b)

Figure 15: Horizontal test results of specimen B7. (a) Force-displacement in $x$ direction. (b) Force-displacement in $y$ direction.

TABLE 3: Test results of specimens with $r=1$.

\begin{tabular}{|c|c|c|c|c|c|c|c|}
\hline \multirow{2}{*}{ Specimen } & \multicolumn{3}{|c|}{ Vertical stiffness $(\mathrm{kN} / \mathrm{mm})$} & \multicolumn{4}{|c|}{ Horizontal stiffness $(\mathrm{kN} / \mathrm{mm})$} \\
\hline & $K_{\mathrm{v} . \exp }$ & $K_{\text {v.ana }}($ error $)$ & $K_{\mathrm{v} . \operatorname{sim}}($ error) & $\sigma_{z}(\mathrm{MPa})$ & $K_{\text {H.exp }}$ & $K_{\text {H.ana }}($ error $)$ & $K_{\text {H.sim }}$ (error) \\
\hline B1 & 271 & $255(-5.8 \%)$ & $249(-8.3 \%)$ & 12 & 0.367 & $0.337(\mathbf{- 8 . 2 \% )}$ & $0.341(-7.1 \%)$ \\
\hline $\mathrm{B} 2$ & 205 & $208(1.5 \%)$ & $202(-1.4 \%)$ & 15 & 0.163 & $0.159(-2.5 \%)$ & $0.166(1.8 \%)$ \\
\hline B3 & 373 & $350(-6.1 \%)$ & $343(-8.1 \%)$ & 10 & 0.371 & $0.371(0.0 \%)$ & $0.373(0.5 \%)$ \\
\hline B4 & 432 & $436(0.9 \%)$ & $406(-6.0 \%)$ & 15 & 0.514 & $0.552(7.4 \%)$ & $0.555(\mathbf{8 . 0 \%})$ \\
\hline B5 & 304 & $278(-8.7 \%)$ & $268(-11.7 \%)$ & 5 & 0.461 & $0.469(1.7 \%)$ & $0.470(2.0 \%)$ \\
\hline
\end{tabular}

TABLE 4: Test results of specimens with $r=2$.

\begin{tabular}{lccccc}
\hline \multirow{2}{*}{ Specimen } & \multicolumn{3}{c}{ Vertical stiffness $(\mathrm{kN} / \mathrm{mm})$} & \multicolumn{2}{c}{ Horizontal stiffness $(\mathrm{kN} / \mathrm{mm})$} \\
& $K_{\text {v.exp }}$ & $K_{\text {v.ana }}($ relative difference) & $\sigma_{\mathrm{v}}(\mathrm{MPa})$ & $K_{\text {H.exp }}$ & $K_{\text {H.ana }}($ relative difference) \\
\hline B6 & 450 & $474(\mathbf{5 . 3 \%})$ & 3 & 1.000 & $0.980(-2.0 \%)$ \\
B7 & 796 & $817(2.6 \%)$ & 15 & 0.697 & $0.670(-3.9 \%)$ \\
B8 & 1032 & $1014(-1.8 \%)$ & 15 & 0.994 & $1.130(\mathbf{1 1 . 7 \% )}$ \\
\hline
\end{tabular}

proposed solutions of compression and bending modulus can be used to calculate the vertical and horizontal stiffness of this type of isolators.

(2) Simplified solutions for compression and bending modulus are derived by a method of weighted residuals. The accuracy is controlled by the selection of trial function of pressure $p$. For square isolators, the error of simplified solution is less than $10 \%$ for compression modulus when dimensionless parameter $\gamma$ is less than 2.6 and for bending modulus when $\gamma$ is less than 3.4. The applicable range covers most types of fiber-reinforced plastic plate isolators.

(3) Finite element analysis results show that neglecting the compressibility of rubber overestimates both compression and bending modulus and neglecting the effect of the Poisson ratio underestimates both the moduli. The proposed solutions include both of the effects and have obvious advantage over the solutions by previous papers.

(4) The validity of the proposed solutions is verified by a series of experimental tests. The error of analytical solution for vertical stiffness is less than $8.7 \%$ and for horizontal stiffness is less than $11.7 \%$. The error of simplified solution for vertical stiffness is less than $11.7 \%$ and for horizontal stiffness is less than $8.0 \%$.

\section{Data Availability}

The data used to support the findings of this study are available from the corresponding author upon request.

\section{Conflicts of Interest}

The authors declare that there are no conflicts of interest regarding the publication of this paper.

\section{Acknowledgments}

This work was supported by National Key R\&D Program of China under Grant no. 2017YFC0703600, the Program for Changjiang Scholars under Grant no. IRT13057, in part by program for Innovation Team of Department of Education of Guangdong Province under Grant no. 2016KCXTD016, in part by the Program for Ram City Scholar under Grant no. 1201541630, and Guangdong Special Program under Grant no. 2014TX01C141. 


\section{References}

[1] X. L. Ge, L. X. Zhu, and W. Yu, "Overview of researches on disaster prevention of buildings in village and town," Journal of Natural Disasters, vol. 23, no. 2, pp. 8-14, 2014, in Chinese.

[2] W. L. Cao, Z. Y. Zhou, Q. Wang et al., "Experimental study on base vibration isolation and anti-seismic masonry structure in rural areas by shaking table test," Journal of Vibration and Shock, vol. 30, no. 11, pp. 209-213, 2011, in Chinese.

[3] F. L. Zhou, Vibration Control of Engineering Structures, Seismological Press, Beijing, China, 1997, in Chinese.

[4] J. M. Kelly, "Analysis of fiber-reinforced elastomeric isolators," Journal of Seismology and Earthquake Engineering, vol. 2, no. 1, pp. 19-34, 1999.

[5] J. M. Kelly, "Seismic isolation systems for developing countries," Earthquake Spectra, vol. 18, no. 3, pp. 385-406, 2002.

[6] J. M. Kelly and S. M. Takhirov, "Analytical and experimental study of fiber-reinforced strip isolators," PEER Report, vol. 11, PEER, Berkeley, CA, USA, 2002.

[7] H. Toopchi-Nezhad, M. J. Tait, and R. G. Drysdale, "Testing and modeling of square carbon fiber-reinforced elastomeric seismic isolators," Structural Control and Health Monitoring, vol. 15, no. 6, pp. 876-900, 2010.

[8] J. M. Kelly and A. Calabrese, "Mechanics of fiber reinforced bearings," PEER Report, vol. 2, PEER, Berkeley, CA, USA, 2012.

[9] P. Angeli, G. Russo, and A. Paschini, "Carbon fiber-reinforced rectangular isolators with compressible elastomer: analytical solution for compression and bending," International Journal of Solids and Structures, vol. 50, no. 22-23, pp. 3519-3527, 2013.

[10] M. Pauletta, A. Cortesia, and G. Russo, "Roll-out instability of small size fiber-reinforced elastomeric isolators in unbonded applications," Engineering Structures, vol. 102, pp. 358-368, 2015.

[11] P. Tan, K. Xu, B. Wang, C. Chang, H. Liu, and F. Zhou, "Development and performance evaluation of an innovative low-cost seismic isolator," Science China Technological Sciences, vol. 57, no. 10, pp. 2050-2061, 2014.

[12] K. Xu, P. Tan, B. Wang et al., "Experimental comparative study of both types of novel simple isolators," Earthquake Engineering \& Engineering Dynamics, vol. 34, no. 6, pp. 11-18, 2014, in Chinese.

[13] Y. Rocard, "Note sur le calcul des propriétès élastiques des supports en caoutchouc adhérent," Journal de Physique et le Radium, vol. 8, no. 5, pp. 197-203, 1937.

[14] A. N. Gent and P. B. Lindley, "Compression of bonded rubber blocks," Proceedings of the Institution of Mechanical Engineers, vol. 173 , no. 3, pp. 111-122, 1959.

[15] A. N. Gent and E. A. Meinecke, "Compression, bending, and shear of bonded rubber blocks," Polymer Engineering and Science, vol. 10, no. 1, pp. 48-53, 2010.

[16] C. G. Koh and H. L. Lim, "Analytical solution for compression stiffness of bonded rectangular layers," International Journal of Solids and Structures, vol. 38, no. 3, pp. 445-455, 2001.

[17] H.-C. Tsai and W.-J. Pai, "Simplified stiffness formulae for elastic layers bonded between rigid plates," Engineering Structures, vol. 25, no. 11, pp. 1443-1454, 2003.

[18] J. M. Kelly, "Behavior of multilayered bearings under compression and bending," in Earthquake-Resistant Design with Rubber, pp. 131-159, Springer, London, UK, 1997.

[19] H.-C. Tsai and J. M. Kelly, "Stiffness analysis of fiberreinforced rectangular seismic isolators," Journal of Engineering Mechanics, vol. 128, no. 4, pp. 462-470, 2002.
[20] J. M. Kelly and A. Calabrese, "Analysis of fiber-reinforced elastomeric isolators including stretching of reinforcement and compressibility of elastomer," Ingegneria Sismica, vol. 30, no. 3, pp. 5-16, 2013.

[21] S. Pinarbasi and Y. Mengi, "Elastic layers bonded to flexible reinforcements," International Journal of Solids and Structures, vol. 45, no. 3-4, pp. 794-820, 2008.

[22] C. D. Xu, "Weighted residuals method for solid mechanics," Mechanics in Engineering, vol. 2, no. 4, pp. 12-20, 1980, in Chinese.

[23] H. C. Tsai, "Compression stiffness of circular bearings of laminated elastic material interleaving with flexible reinforcements," International Journal of Solids \& Structures, vol. 43, no. 11-12, pp. 3484-3497, 2006.

[24] J. A. Haringx, "On highly compressible helical springs, and rubber rods, and their application for vibration-free mountings," Philips Research Reports, vol. 4, pp. 49-80, 1949.

[25] Architectural Institute of Japan, Recommendation for the Design of Base Isolated Building, Marozen Corporation, Tokyo, Japan, 1993.

[26] Standardization Administration of the People's Republic of China, GB/T 20688.1-2007: Rubber Bearings-Part 1: Seismic Protection Isolators Test Methods, SAC, Beijing, China, 2007. 


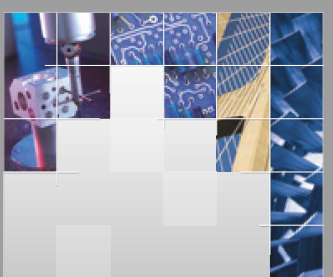

\section{Enfincering}
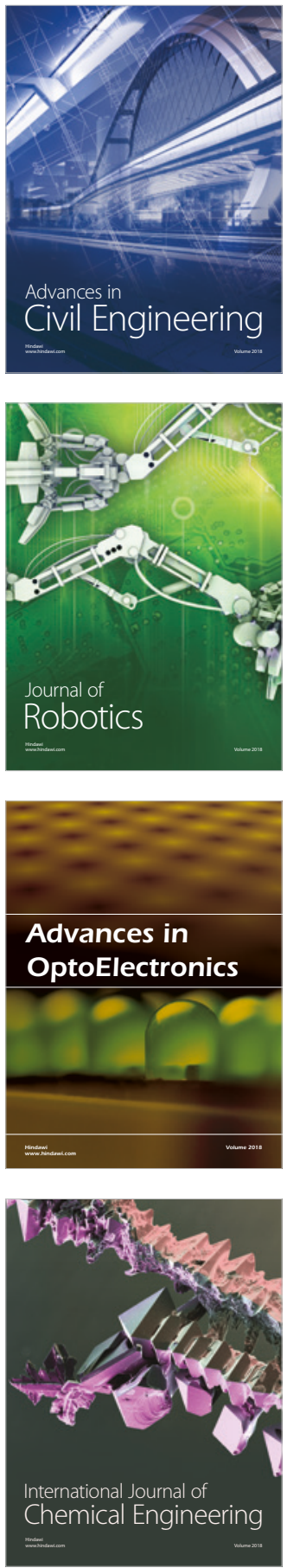

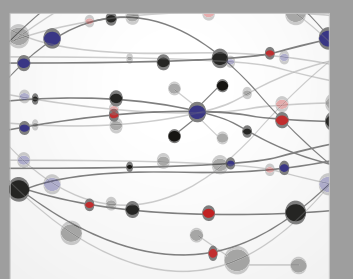

\section{Rotating \\ Machinery}

The Scientific World Journal

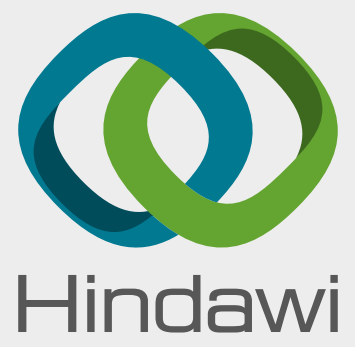

Submit your manuscripts at

www.hindawi.com
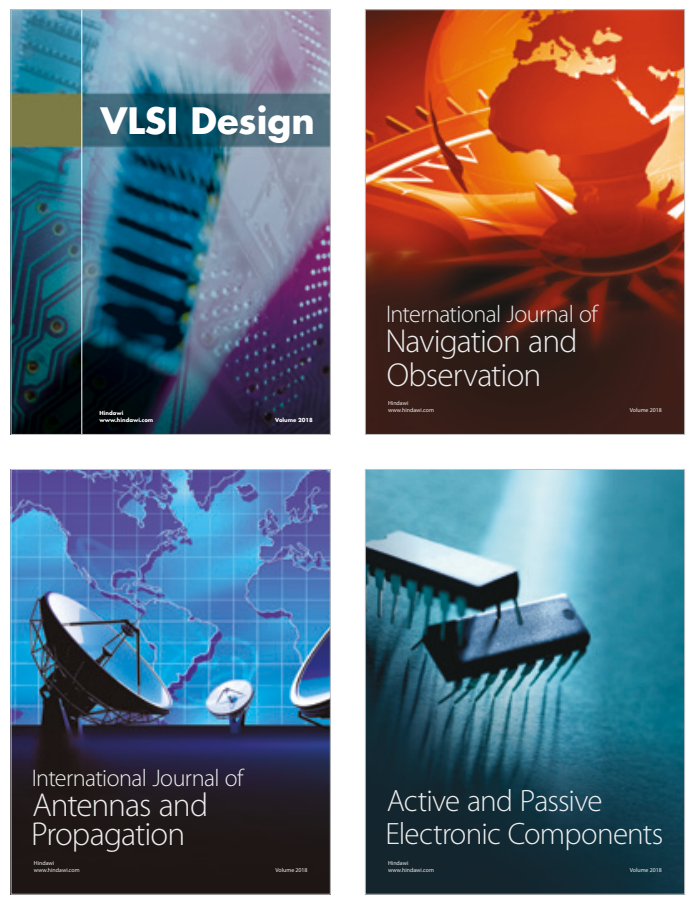
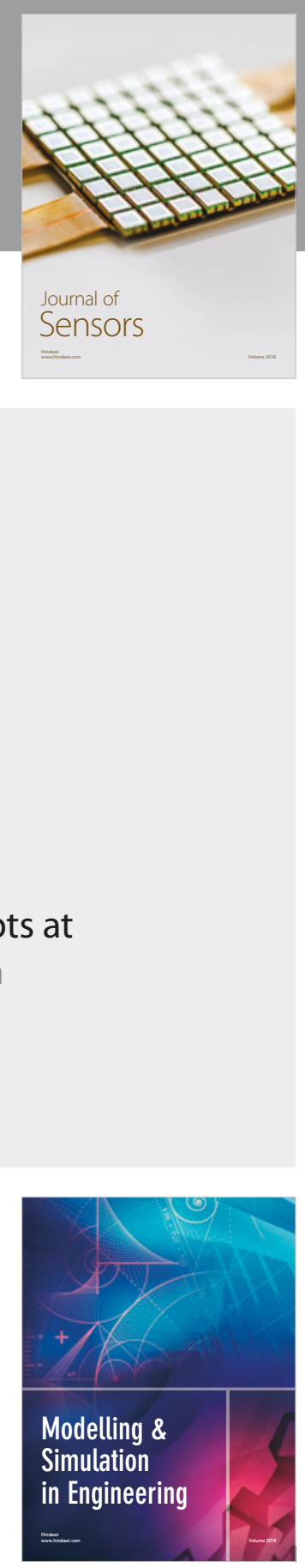

\section{Advances \\ Multimedia}
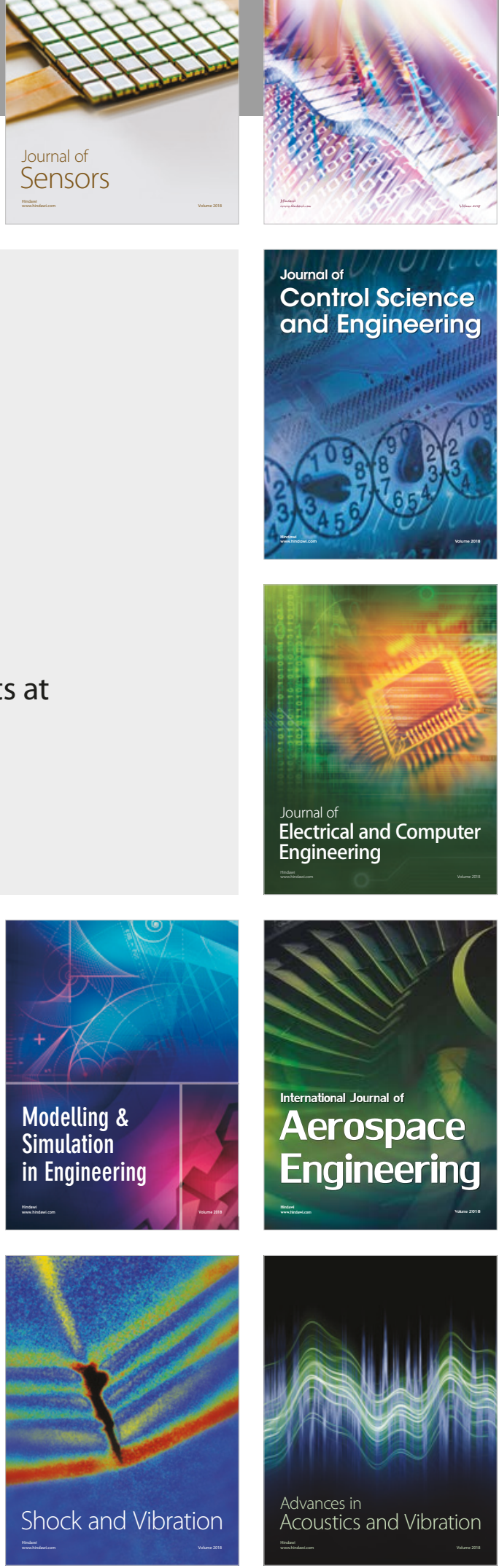